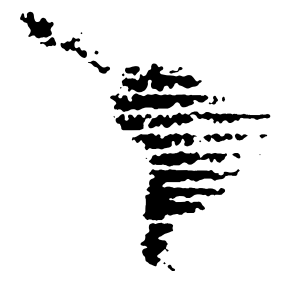

\title{
UNA PROPUESTA PARA LA FUNDAMENTACIÓN TEÓRICA Y FILOSÓFICA DE LA EDUCACIÓN EN DERECHOS HUMANOS
}

\author{
MSc. Juan Carlos Naranjo Segura*
}

\section{Resumen}

El presente artículo tiene como meta plantear una reflexión acerca de la fundamentación teórica y filosófica de la educación en derechos humanos. Para ello, se parte de la comprensión de que los derechos son un escenario de resignificaciones sociales, donde la base para alcanzar los cambios sociales, radica en la posibilidad de que cada persona pueda posicionarse en su propio contexto, lo comprenda e intente cambiarlo cuando este violente o atente contra la dignidad de la persona. Desde esta lógica, se define los derechos humanos como espacios de lucha, en los cuales la educación puede cumplir un papel clave, en cuanto permita la comprensión de la propia realidad. De esta manera, se analizará cómo la Filosofía de la Liberación permite ser un insumo desde el cual se puede alcanzar un humanismo que permite, desde los espacios de la cotidianidad, la vivencia de los valores propios del respeto de los derechos.

Palabras clave: derechos humanos, educación, Filosofía de la Liberación, humanismo.

\section{Abstract}

This article aims to establish a reflection on the theoretical and philosophical foundation of human rights education, to do so, we start from the understanding that rights are a system new social meanings scenario, where the basis for achieving such purpose lies in the possibility that people have to be placed in their own context, to understand it and change it every time this violates or undermines the dignity of the human person. From this logic, human rights are defined as sites of struggle, where education should play a key role, as it must allow an understanding of reality itself. Thus, it will be analyzed how the Philosophy of Liberation becomes an input from where people can reach a humanism that permits to experience values of respect for human rights on everyday life.

Keywords: human rights, education, Philosophy of Liberation, humanism.

\footnotetext{
* Profesor de Estudios Sociales y Educación Cívica en el Liceo Experimental Bilingüe José Figueres Ferrer de Cartago y profesor de la Escuela de Formación Docente de la Universidad de Costa Rica. Máster en Derechos Humanos de niñez y adolescencia de la Universidad de Costa Rica.
} 
Los derechos humanos, como discusión teórica, son un campo que ha ido posicionándose desde varias aristas en la sociedad costarricense. El Estado ha operado, bajo una línea de acción en cuyo discurso se externa en ser garante del respeto de la dignidad humana.

La importancia que se le ha dado a la temática parte del hecho de valorar el papel que el Estado ha tenido en el reconocimiento de derechos a distintos grupos, reconocidos como excluidos, mediante la ratificación de convenciones internacionales, en cuyas aprobaciones se establece una serie de compromisos mediante los cuales se busca el establecimiento de un escenario que permita que todos y todas sean respetados sin distinción alguna.

Es bajo esa perspectiva que se puede comprender cómo este nuevo posicionamiento teórico, denominado enfoque de derechos, paulatinamente ha empezado a vislumbrase en distintos ámbitos de la sociedad costarricense. Uno de esos sectores es el caso del ámbito educativo. La incorporación de los derechos humanos como un contenido por desarrollar en disciplinas como en Estudios Sociales y Educación Cívica es un ejemplo de este argumento. Definirlos como ejes transversales de la educación costarricense es otro de los medios que explica cómo empiezan a fundarse nuevos escenarios de discusión bajo dicho enfoque.

Dentro de estas disertaciones, el objetivo del presente artículo adquiere un sentido, ya que se busca establecer una reflexión acerca de la fundamentación teórica y filosófica de la educación en derechos humanos, que permita el fortalecimiento de los derechos humanos de las personas menores de edad.

Para fundamentar dicha reflexión, se parte de la comprensión de que la garantía de los derechos humanos de las personas menores de edad alcanza sentido a través de un modelo educativo que permita desarrollar en el grupo de estudiantes la aptitud de la criticidad de su propia realidad, de su condición como sujetos de derechos, para que, partiendo de ese conocimiento, puedan convertirse en personas con la capacidad de transformar aquellos escenarios que atentan contra el respeto de su dignidad.

Para poder cumplir con el objetivo planteado, el presente artículo desarrolla tres ejes temáticos. El primero de ellos corresponde a una reflexión teórica acerca del concepto de los derechos humanos, la cual busca ubicarlos como fruto del proceso histórico que emana de la lucha del ser humano por alcanzar mejores condiciones de vida. De la misma manera, se les visualiza como parte de un contexto, que para nuestro caso coincide con el fenómeno cultural llamado globalización, una realidad enmarcada por condiciones que ponen en riesgo el respeto de la dignidad de la persona, de ahí la necesidad de comprender que los derechos se convierten en la base de las luchas que debemos asumir para su garantía. 
El segundo eje temático profundiza en el sustento filosófico que debe tener el trabajo en derechos humanos. En este punto, se acudirá a la corriente teórica de la Filosofía de la Liberación (en adelante FL), la cual es concebida no solo como un corpus teórico, sino como un modo de vida, a través del cual cada quien se asume como un sujeto de derechos, replanteando su significado y el de los demás como iguales a sí mismo.

Finalmente, en el tercer eje, se concluye al respecto de la relación que se establece entre estos cuerpos teóricos. Los derechos humanos se pueden fortalecer desarrollando un humanismo desde la perspectiva de la Filosofía de la Liberación, la cual se convierte en un insumo que permite establecer una relectura del sistema educativo costarricense, desde la perspectiva de la opción preferencial por los oprimidos, en donde se busque develar la situación de los derechos del conjunto de estudiantes y, de esta forma, analizar las condiciones reales sobre las cuales se podrían dar las bases para el fortalecimiento de los derechos humanos.

\section{Un acercamiento al concepto de los Derechos Humanos de la Niñez y Adolescencia}

El concepto de derechos humanos teóricamente ha tenido diferentes acepciones para su abordaje. En algunos casos, se les relaciona con un conjunto de leyes o normas que los Estados asumen, en función de la protección de la dignidad de las personas, sin que medie ningún tipo de condición especial. Por esto, las Naciones Unidas, al declarar los derechos humanos, estableció en función de ellos que:

Toda persona tiene los derechos y libertades proclamados en esta Declaración, sin distinción alguna de raza, color, sexo, idioma, religión, opinión política o de cualquier otra índole, origen nacional o social, posición económica, nacimiento o cualquier otra condición. (ONU, artículo 2, 1948).

La misma Organización de Naciones Unidas (1948) los define como aquellas libertades, facultades y reivindicaciones relativas a bienes primarios o básicos que incluyen a toda persona, por el simple hecho de su condición humana, y que garantizan la tenencia de una vida digna.

No obstante, a la hora de profundizar en su disección, se puede sintetizar dos corrientes o posiciones teóricas, que se describen a continuación:

Una de ellas es la iuspositivista, cuya fuente de acción es el derecho positivo, en donde según lo argumentado por Orozco (s.f.), no se rechaza la existencia de ciertos derechos de tipo naturalista, es decir, que son innatos del ser humano y que su existencia es independiente del reconocimiento del Estado, pero sostiene que tales "derechos" son valores morales y no auténticos derechos, jurídicamente considerados, de ahí el valor que adquieren por ser un marco legal para la convivencia en sociedad. 
El principal argumento dentro de esta visión, es que los límites y la jerarquía de los derechos humanos dependen de su reconocimiento en el marco jurídico y político que regula y protege al Estado. Esto implica que los derechos subjetivos requieren verificarse en términos de lo que determinadas normas jurídicas disponen. Orozco (s.f.) afirma que para los positivistas, los llamados "derechos humanos" tienen un carácter primordialmente moral, sin prejuicio de que pueda haber derechos jurídicos correlativos en los sistemas jurídicos positivos, ya sea de carácter internacional o alguno estatal, pero rechaza que el derecho exista sin el reconocimiento dentro del marco jurídico de los Estados.

Por otro lado, se encuentra el iusnaturalismo, una corriente inspirada en la idea de la ley de la naturaleza humana, que postula la existencia de ciertos derechos innatos al ser humano cuya validez era independiente a los sistemas positivos. Esta se basa en el siguiente principio:

Hay ciertos principios morales y de justicia universalmente válidos, los cuales pueden ser conocidos a través de la razón humana, y en el caso de que algún sistema o norma no se adecuen a tales principios universales, los mismos no pueden ser considerados como jurídicos. (Orozco, s.f., p. 30).

Dentro de esta visión, por encima del derecho positivo, mutable e imperfecto, hay un derecho natural de carácter universal, el cual constituye el auténtico derecho en cuanto corresponde a facultades y poderes innatos del ser humano. Los derechos humanos, según esta escuela, corresponden a este conjunto de derechos naturales que el derecho positivo debe reconocer y reglamentar su ejercicio.

Bajo las líneas teóricas descritas anteriormente, el presente artículo, para comprender el significado de los derechos humanos, apuesta por una línea en donde se analizan desde su dimensión más histórica, trascendiendo con ello su expresión legalista y naturalista, en tanto son analizados más como fruto de las distintas luchas sociales acaecidas a lo largo del tiempo'. De tal forma, a la hora de hacer un abordaje crítico de su significado, se debe comprender que en su esencia radica la construcción y el rompimiento de paradigmas, en donde el posicionamiento de distintos grupos por mejorar sus condiciones de vida es el germen para dar pie a nuevas resignificaciones de derechos, lo cual contribuye a la transformación y mejoramiento de la sociedad.

La noción histórica sobre los derechos universales, según Arrollo (2009), parte de la idea de que son condiciones inalienables del ser humano; sin embargo, este elemento determinante

1 Según la profesora Roxana Arrollo (2009), los derechos humanos deben de ser comprendidos en su dimensión histórica como fruto de las luchas de los distintos grupos por alcanzar su dignidad, luchas que son reconocidas y legitimadas por los estados a través de la elaboración de instrumentos legales para su cumplimiento y protección. 
en la concepción de dicha categoría ha provocado múltiples dilemas, ya que implica la dificultad misma de definir qué es lo humano. El término derecho establece el principio fundamental de la esencia humana: la igualdad, entendida como la no discriminación. No obstante, esa percepción de "igualdad" debe ser analizada por el elemento histórico de las distintas ideologías desde las cuales se construyó. Así entendido, se debe comprender que lo "humano" es fruto de una edificación de determinados grupos.

De esta forma, el análisis de los derechos implica observar los distintos panoramas desde donde existen grupos marginados e históricamente excluidos. Se trata, entonces, de abordarlos desde una perspectiva ampliada, a través del tamiz de la condición etaria, del género, la raza, la nacionalidad, entre otros componentes esenciales sobre los cuales se han fundado históricamente la negación de derechos.

Esta lógica de entender los derechos humanos permite visualizarlos como un cuestionamiento de orden social. Esto implica enraizarlos como parte del entramado de las relaciones, en donde existe un juego de poder, que se expresa en las tensiones surgidas por la predominancia de unos grupos que se imponen sobre otros. Estas tensiones emanan cuando los desposeídos van abriendo espacios para hacer valer sus derechos y, con ello, construyen resignificaciones que a su vez van transformando poco a poco la cultura, tal y como lo expresa la profesora Arrollo (2009) en su ponencia sobre los derechos humanos: (...) transformaciones culturales, transformaciones identitarias, esto porque permite crear sujetos políticos. Transformaciones en las relaciones de poder: para crear condiciones de igualdad, para la democracia y para la ciudadanía.

Los distintos procesos en la historia han conllevado a que el concepto de los derechos humanos no esté ligado a la homogeneidad, sino más bien a la diversidad. La argumentación del porqué es necesario aclarar tal distinción parte de la misma contradicción entre los derechos estipulados en la Declaración Universal de Derechos Humanos, como principios para todos, ya que lo propuesto en la teoría, en la realidad no se aplicó. Así, en la práctica, los derechos humanos surgen teniendo una serie de condicionantes de género, raza, edad y ubicación espacial, que provocan condiciones de agresión de derechos sobre muchos grupos que quedaron al margen.

Las distintas luchas realizadas por aquellos que han sido marginados de derechos van creando nuevos significados hacia eso que entendemos por derechos. Esto conduce, a su vez, a nuevos posicionamientos jurídicos, que buscan el reconocimiento y la protección por parte de los estados. Las Convenciones Internacionales son un ejemplo de lo anterior. Mediante ellas, se reconocen jurídicamente los derechos de una población específica, 
al tiempo que se enseña al resto de la humanidad que dicho grupo es sujeto de derechos. Este es el caso de lo que pasó con los niños y las niñas, los y las adolescentes, las mujeres, los pueblos indígenas, las personas con condiciones especiales, las personas migrantes, entre otros grupos.

Esta noción de luchas sociales y de resignificaciones es lo que lleva a entender los derechos humanos como un medio de protección del ser humano, esto a partir, ahora sí, del marco jurídico y ético de la sociedad que los estipula. Desde esa misma lógica, son estándares de medición para el buen funcionamiento de los Estados, ya que son entes reguladores de los gobiernos.

Es bajo esta forma de entender los derechos humanos que se debe colocar en discusión el tema de las personas menores de edad, ya que es en 1998, a partir de la promulgación en Costa Rica del Código de la Niñez y la Adolescencia, cuando empieza a generarse un proceso de cambio, en la medida en que pasan de ser objetos a ser sujetos de derechos, tal y como se expresa Fernández (2007):

La Declaración de los Derechos Humanos y la Convención Internacional sobre los Derechos del Niño (CDN) impulsan una revolución simbólica, un cambio de paradigma respecto del lugar, los derechos y las garantías de la infancia. $\mathrm{La} C D N$ reconoce a los niños/ as como sujetos sociales y sujetos de derechos, removiendo al ideal jurídico social de la situación irregular (p. 2).
Lo anterior implicó que los adultos están a cargo de su desarrollo integral y el Estado asume el deber y la obligación de garantizarles condiciones para su desarrollo y la satisfacción de sus necesidades.

Junto con la discusión anterior, se encuentra la interpretación de los menores de edad como sujetos integrales. Esta afirmación, que se expone de manera simple, implicó toda una transformación en la forma de vivenciar los derechos humanos de esta población. Esto permite trascender la percepción de la niñez enfocada en la doctrina de la situación irregular, en donde son percibidos como seres incompletos, sin capacidad ni derecho, en donde la obediencia y la sumisión a la autoridad del adulto era la norma. Así, Fanlo (s.f.) explica el cambio en el paradigma, y

(...) junto con el mérito de constituir un cambio fundamental de paradigma, la postulación de una doctrina de protección integral de los derechos de la infancia que, al referirse a algunos instrumentos jurídicos internacionales (en concreto a los derechos humanos), habrían promovido un notable salto cualitativo en la consideración jurídica y social del niño (p. 161).

De la misma manera, los derechos humanos responden a un contexto histórico concreto que los determina y los convierte en una necesidad para la convivencia humana, esto significa que el enfoque de derechos como tal, no es una teoría de hoy, sino que ha estado 
presente en otras coyunturas. Por tanto, la manera en que se les conciba dentro de su comprensión histórica debe de arraigarse en medio del modelo de sociedad en que están inmersos.

Bajo esta línea de acción, vale la pena establecer una reflexión del contexto que nos rodea, que determina dicha noción de derechos. La humanidad en las últimas décadas ha sido influenciada por los ideales capitalistas de consumo, acumulación, avaricia e individualismo, los cuales están ocasionando el aumento de la pobreza como fruto de las desigualdades sociales que se han potenciado, aspectos que se convierten en la principal manifestación del orden imperante. Es bajo el marco del llamado mundo globalizado, en donde se tiene la tendencia, a la violación y negación de derechos, ya que es una realidad caracterizada por el deshumanismo, que emerge por la forma de vida de los hombres y mujeres, tal y como lo expresan Alarcón y Molina (2008):

Porque si estas sociedades deshumanizadas son, obviamente, una construcción social, producto de los hombres y las mujeres que las componen; también las mujeres y los hombres tenemos la absoluta posibilidad ( $i y$ la obligación?), aún en condiciones marcadamente subordinadas pero no definitivamente ineluctables, de construir otro tipo de sociedades, basadas en relaciones de solidaridad, fraternidad y equidad (p. 38).
Cada día el rico se vuelve más rico, mientras que los pobres pasan el umbral de la marginalidad y el olvido. Los ricos cada vez son menos cantidad, mientras que los pobres en el mundo son una cifra que no se puede ni cuantificar con claridad. De ahí que Souza (2004) exprese una serie de contradicciones que describen la realidad en la que vivimos, la cual estipula el enfrentamiento dialéctico entre los intereses del orden mundial de la época del industrialismo, que se debilita de forma irreversible a partir de las contradicciones que se explican con las siguientes premisas:

- $\quad$ El mundo es puntualizado como un mercado constituido por economías sin sociedades, es decir, un mundo en donde ya no importan las sociedades.

- La globalización es vista como reemplazo de los fines por los medios, es decir, los valores que establece la doctrina neoliberal imperante son crecer, crecer, crecer y exportar, en donde la mano invencible del mercado cuida de la distribución de los beneficios del crecimiento económico, esto acompañado del reemplazo de la razón del Estado, por la razón del mercado, el cual se pregona como "libre" pero que no es tan libre.

- La vulnerabilidad del capital y la vulnerabilidad del trabajo implica nuevas relaciones laborales caracterizadas por la flexibilidad laboral y el fin del contrato social entre el capital y el 
trabajo, donde el desempleo y el subempleo se han transformado en alternativas para solucionar los problemas de los inversionistas transnacionales y banqueros nacionales. Lo anterior está unido al otro contraste que se expone, el cual se describe como el desempleo tecnológico como fuente de lucro, en donde la clase trabajadora se abandona para darle paso a la tecnología.

- El sector público como fuente de problemas y el sector privado como fuente de soluciones, en donde se busca destruir la ideología del Estado, para establecer a la ideología del mercado, para ello se visualiza lo público como sinónimo de ineficiencia, corrupción e incompetencia, mientras lo privado es pintado de colores contrarios de eficiencia, seriedad y competencia, esto conlleva a la paradójica realidad de la presencia de un "buen gobierno" para los actores transnacionales y "mal gobierno" para la sociedad nacional, en donde prevalece una democracia representativa, donde los electos no deciden y los que deciden no son electos, porque corresponde al gobierno de los organismos transnacionales, de ahí que se visualice también como contradicción, al individualismo que es promovido por la competitividad y la solidaridad requerida por la sostenibilidad, lo cual está llevando a un darwinismo social.

- El crecimiento económico con exclusión social, que es la consecuencia de todo lo anterior, en donde nunca antes en la historia de la humanidad había existido tanta riqueza acumulada en tan pocas manos, mientras la mayor parte de la población vive en la absoluta miseria. Esto va unido a la lógica de un mundo que pregona una ética en medio de un enorme vacío de valores, la presencia de una ciencia sin conciencia que es usada para el lucro de la sociedad o el trabajo con los pobres sin manejar el concepto de la pobreza, de ahí que su principal manifestación sea la presencia de un mundo de los excluidos en la era del acceso o el hambre en un mundo de abundancia, prisioneros de la opulencia y los prisioneros del desamparo, o aquellos que se convierten en vendedores de ilusiones en medio de una gran mayoría que se convierten en los huérfanos de la esperanza, en un mundo que propone un pensamiento único, abandonando el pensamiento crítico.

La explicación a las contradicciones permite entender la lógica del modelo económico y la forma en que se hace política, en donde se propicia precisamente el desarrollo de la pobreza como la madre de todos los males en nuestros días. La explicación a esta realidad en 
dos palabras es: neoliberalismo y globalización. Estos elementos han transformado toda la realidad vivida por los sujetos, tal y como lo describen Martínez y Agüero (s.f.): "La globalización amplió y profundizó la desigualdad social, fragmentó y mercantilizó las relaciones sociales, aceleró la pérdida de ciudadanía y transformó el futuro en una amenaza aterradora para los sujetos sociales y para las sociedades que estos integran" (p. 1).

Ambos conceptos son las caras de una misma moneda, que incluso están unificadas por un modelo de producción atroz, en donde el capital se impone sobre el trabajo. En otras palabras, la búsqueda desmedida por la acumulación de la riqueza, se convierten en el contexto, tanto a nivel local, nacional e internacional, constituyentes del mundo desde donde se deben desarrollar los derechos humanos.

El neoliberalismo es entendido para muchos autores como el principal causante de la crisis global, en las dimensiones económica, social, alimentaria y ambiental, a la cual hoy está sujeta la humanidad. Esa crisis conforma la coyuntura que presiona y da sustento a los derechos humanos desde su dimensión histórica, pues implica, desde el presente marco, su comprensión como un espacio de lucha, de emancipación y de liberación de la opresión causada por ese modelo económico que arraiga la violación de la dignidad humana.

Como se ha tratado de sostener, los derechos humanos han conformado una realidad que ha marcado a la humanidad en diferentes épocas, constituyéndose en un campo de lucha social e ideológica para la emancipación humana. Esta forma de entenderlos permite trascender su concepción legal ya que los concibe como un espacio para la resignificación del ser humano y su dignidad. El filósofo de derecho, Joaquín Herrera Flores (2000, s.p.), lo ha expresado con claridad al definirlos como “(...) el conjunto de procesos (normativos, institucionales y sociales) que abren y consolidan espacios de lucha por la dignidad humana".

Entendidos de esta manera, los derechos humanos, en el contexto presente, deben ser abordados no como un producto acabado dentro de la construcción histórica, sino como un punto para la comprensión de la relación entre el capital y el trabajo. En esta relación, el capital va a buscar las condiciones para poder desarrollarse, creando un contexto histórico que violenta la dignidad, todo esto por medio de un proceso de socialización, a través del cual, las instituciones como la familia y la escuela son clave, ya que es por medio de ellas que el mercado va moldeando a los grupos sociales en su cotidianidad, ubicando su función dentro de la estructura social. Así, De Lemos (2007) plantea:

Tal compreensão revela o resultado da eficácia de instituições como o Estado e o mercado, que a través de procesos de socialização homogênea, delineiam para os grupos e agentes os 
modos de ser e agir cotidianamente. Socialização que busca moldar a consciência de cada agente e definir o lugar que devem ocupar em cada espaço social2 (p. 13).

El análisis que se puede realizar de los derechos humanos dentro de su marco contextual implica un espacio de crítica del modelo de sociedad imperante y de las relaciones de poder presentes dentro de ella. Al convertirse en crítica, el enfoque de derechos va más allá de la visión positivista y naturalista de los derechos humanos, y se puede interpretar como un marco referencial de análisis que permite la búsqueda del respeto de la dignidad, cuya consecuencia es la liberación de toda forma de opresión, en donde los pueblos luchan por crear justicia social y hacen valer su condición de persona en sus múltiples dimensiones. Tal y como afirma Aguiló (2009): "La principal contribución de los pueblos a la cultura de los derechos humanos es su lucha por la dignidad y la diversidad humanas en sus múltiples versiones y lenguajes, que es, en el fondo, la lucha por la justicia social y cognitiva" (p. 202).

La cita anterior expone la interpretación sobre que los derechos humanos, en medio de un contexto capitalista y

2 "Tal entendimiento revela el resultado de la eficacia de las instituciones como el estado y el mercado, que a través de procesos de socialización desde lo homogéneo, permite ubicar a los grupos y su maneras de ser y de actuar sobre una base diaria. Socialización que pretende formar la conciencia de cada agente y establecer el lugar debe ocupar en cada espacio social" (traducción propia). neoliberal, se convierten en un espacio para transformar la realidad social y un recurso para que los pueblos logren justicia sin distinción alguna. Tal afirmación conlleva a entender la fundamentación de que dichos derechos no son solo una fuente de análisis de las condiciones de vida de las personas, sino que son un camino para cambiar las situaciones de injusticia y violación a las cuales es sometido el ser humano de hoy.

\section{Fundamento filosófico de la educación en derechos humanos}

En relación directa con esa noción acerca de los derechos humanos, es pertinente establecer la base filosófica desde la cual se establecen los escenarios para el desarrollo de un humanismo que permite, desde los espacios de la cotidianidad, la vivencia de los valores propios del respeto de los derechos. En este sentido, la Filosofía de la Liberación da un compendio de ideas vinculadas con esta finalidad, tal y como se expresa a continuación:

Sobre la base de las reflexiones anteriores, Ellacuría plantea la necesidad del aporte de la filosofía, y de otras disciplinas, especialmente en el fomento y provocación de una conciencia colectiva, que posibilite la transformación, y en la creación de nuevos modelos económicos, políticos y culturales que hagan posible la civilización de la pobreza. (...) la cual rechaza la acumulación del capital como motor de la historia y la posesión-disfrute de la riqueza como principio de humanización, y 
hace de la satisfacción universal de las necesidades básicas el principio del desarrollo y del acrecentamiento de la solidaridad compartida el fundamento de la humanización. (Samaur, s.f., s.p.).

Retomando el texto anterior, se puede sintetizar que la base central de la Filosofía de la Liberación radica en dos ideas centrales: el rechazo de las condiciones estructurales que violentan la dignidad humana, a la vez que pregona la liberación, la cual debe ser entendida como la búsqueda de la transformación de la sociedad, retomando un modelo más humano de convivencia.

Si bien es cierto hasta este punto del análisis se hace necesario el abordaje de la conceptualización de filosofía y su función, más importante es replantear el aporte que da el acto del filosofar a la reflexión de los derechos humanos. Como un conjunto de saberes, como modo de vida y como explicación de la realidad social, la filosofía le brinda al profesional en derechos humanos un sustento a su labor. El vínculo entre filosofía y derechos humanos implica convertirlos en un modo de vida, a través del cual cada quien se asume como sujeto de derechos, reconociendo a su vez a los demás como iguales.

Para lograr eso, es fundamental que el especialista en derechos humanos asuma su realidad histórica en donde, sin duda, es necesaria la filosofía, pues permite actualizar a la inteligencia como estructuralmente dinámica y compleja, para luego volver a la realidad buscando transformarla. En esto consiste el sentido primordial de la función liberadora que ella posee. Por eso, la teoría no se contrapone a la práctica, por el contrario, se debe utilizar como el medio para lograr la liberación de los oprimidos. Es decir, la teoría es usada para comprender los fenómenos sociales acaecidos en la realidad y que marginan a ciertos grupos. En ese sentido, la educación en derechos humanos debe asumir, como meta fundamental, que su conocimiento debe ser usado para apoyar teóricamente el cumplimiento, la garantía y el fortalecimiento de los derechos de las personas.

Un principio básico para la interpretación de la Filosofía de la Liberación es el reconocimiento de la realidad social de un grupo simbólico de la humanidad, que son los excluidos. Hacia ellos y ellas es que se dirige toda la acción del filosofar, como su opción liberadora. De esta manera, el enfoque de derechos reconoce en las figuras de niños, niñas y adolescentes, sujetos que históricamente han sido oprimidos, por eso se debe de partir de dicha realidad para buscar su transformación, asumiendo una función crítica y creadora. Tal y como lo expone Mora (2006, p.181): "La finalidad principal de la Filosofía estará, pues, en la liberación de las mayorías oprimidas. Ese es, en cierto modo, el horizonte fundamental del quehacer filosófico en su función crítica y creadora".

De esta forma, el actuar a partir de los derechos humanos con una base teórica desde la Filosofía de la Liberación 
implica no solo dar un aporte desde la teoría, sino desde la praxis, a la transformación de un modelo de sociedad que ve en la niñez y la adolescencia una población débil y carente de derechos. Además, revela la vigencia de la filosofía como saber, como orientación de la conciencia individual o personal, colectiva o social y política; como forma de vida, síntesis de vida intelectual activa, y como opción de una búsqueda de humanidad y no de opresión o de sumisión. Es a partir de esta visión que se plantea la necesidad de la Filosofía de la Liberación dentro de un marco de educación en Derechos Humanos de la Niñez y la Adolescencia.

\section{Humanismo: un acercamiento a la} educación en derechos humanos

(...) el verdadero humanismo tiene dos vertientes, una hacia la vida social y desde ese punto de vista mi existencia estará comprometida en gran parte con los demás a través de las instituciones, y la otra se refiere a mí mismo, con lo que me permite comprometerme conmigo mismo. (Lukács, 2000, p. 9).

Como actividad propia del ser humano, el acto del filosofar da por sí solo un aporte valioso a su esencia, que es la posibilidad de vivir a plenitud nuestra condición humana. Sin embargo, como seres sociales que somos, esto se convierte en un compromiso con los demás, en cuanto abre la posibilidad de acercarnos los unos con los otros en igualdad de condiciones, elemento básico que requiere la educación en derechos humanos, sobre todo si se trata de niñez y adolescencia, pues se requiere un mayor nivel de sensibilidad que permita reconocerlos como seres de derechos.

Desde el plano individual, la filosofía es un compromiso con uno mismo, en donde toda acción vivida adquiere significación cuando se es capaz de dar respuesta a los problemas elementales de la existencia. En ese sentido, las aplicaciones concretas de la filosofía como un sustento a la educación en Derechos Humanos de la Niñez y la Adolescencia radican en el hecho mismo de que el enfoque de derechos debe permitir una práctica de vida, en donde con cada acto realizado se busque poner en práctica tal teoría.

Por último, la actitud crítica que permite desarrollar en el ser humano el acto de filosofar establece la capacidad de interrogarse el porqué de las cosas, lo cual se convierte en el motor para lograr, a través del proceso educativo, un conocimiento, en donde el análisis de la realidad es vital para poder convertir estos dos elementos (filosofía y derechos humanos) en herramientas que busquen alcanzar, paulatinamente, la liberación de los grupos oprimidos, en este caso la niñez y la adolescencia.

Así entendida, la Filosofía de la Liberación es más que un conjunto de pensamientos del ser humano a lo largo del tiempo, pues son conocimientos que deben de ser aplicados en la práctica de 
la vida en sociedad, que llevan consigo a un compromiso de cada quien para la transformación de aquellas esferas personales y sociales que producen una violación de los derechos de las personas.

Desde este enfoque, los derechos humanos deben ser concebidos más allá de un absoluto. Por el hecho de su existencia, desde la Declaración Universal de Derechos Humanos en 1948, deben establecerse como espacios en construcción, de lucha y garantía, que desde la vida deben de constituirse en un sitio para la práctica de la liberación a favor de todas y todos aquellos que son oprimidos; es decir, los derechos humanos deben ser escenarios de lucha por la liberación y la transformación de las contradicciones que potencian el irrespeto de la dignidad humana, una humanidad que no es concebida teóricamente, sino desde su realidad histórica y social.

\section{A modo de conclusión: hacia una educación que permita el fortalecimiento de los derechos humanos de las personas menores de edad}

Dentro de la lógica de la Filosofía de la Liberación existen categorías centrales que explican por qué se hace uso de este referente filosófico como un medio para desarrollar un modelo de educación que permita el fortalecimiento de los derechos de las personas menores de edad. Uno de esos elementos fundamentales, que trasciende a tal reflexión, tiene que ver con el concepto de libertad, la cual es definida a partir de la opresión y la marginalidad. Dussel (1972) la define de la siguiente manera:

La libertad concebida no como el poder mágico que instaure un orden de cosas, ideal y perfecto, mediante la negación y el repudio de una realidad presente sino, por el contrario, comprendiéndola en su verdadero significado, como la admisión a una realidad concreta y determinada para transformarla a través de la acción de los hombres que en ella se encuentran, viviéndola y asumiéndola críticamente (p. 17).

Entendida de esa manera, la libertad humana es una cualidad que tiene que ver con el ser social, que implica asumirlo, reconocerlo e insertarse críticamente en su seno para transformarlo. La libertad posibilita que el ser humano se involucre creativa y constructivamente en el proceso histórico orientado a lograr liberación. Así, Dussel (1972) plantea que

(...) de este modo, la libertad deviene la capacidad de practicar actos libres, que a su vez son liberadores, transformándolos así en el poder de liberar (...) La libertad existe, el problema no radica en la discusión bizantina de su significado abstracto, sino en establecer en qué consiste, cuáles son las condiciones para que pueda ser ejecutada y qué resultados conduce (p. 26).

La comprensión del concepto de libertad permite establecer un vínculo muy directo con el proceso educativo, el 
cual debe ser percibido como un acto de filosofar, esto en la medida de que es un compromiso por desideologizar el sistema mismo en que se desarrolla, develar la verdad sobre su aporte al fortalecimiento de los derechos de los y las adolescentes que son formados a través de él.

La educación crítica emerge como una herramienta para lograr tal fin, ya que parte del reconocimiento del contexto inmediato, que emana con una serie de contradicciones que violentan, de forma estructural, el respeto de los derechos de las personas. Una educación en derechos humanos requiere de un enfoque teórico y filosófico, que permita develar esos escenarios de injusticia y marginación, esto debería conducir a una profunda insatisfacción que evoca hacia una voluntad de compromiso por la transformación de toda forma de discriminación.

No hay educación liberadora si no pensamos que hay algo de que liberarse y no hay educación transformadora si no se siente un deseo y una posibilidad de cambio social. No es necesario estar de acuerdo en un mismo modelo ideal, ni siquiera tener una alternativa global ya diseñada, sino compartir una orientación utópica para superar las limitaciones del presente y creer que la educación no puede ni debe rehuir sus responsabilidades.

Partiendo del modelo de una educación crítica y liberadora es que se desarrolla el análisis de las posibilidades de la educación como un medio para el desarrollo de un humanismo que permita el respeto de los derechos de las personas. Según Peter McLaren (1997), la pedagogía crítica invita a analizar la relación entre experiencia, conocimiento y orden social, con una perspectiva transformadora:

Todo el proyecto de la pedagogía crítica está dirigido a invitar a los estudiantes y a los profesores a analizar la relación entre sus propias experiencias cotidianas, sus prácticas pedagógicas de aula, los conocimientos que producen, y las disposiciones sociales, culturales y económicas del orden social en general (...) La pedagogía crítica se ocupa de ayudar a los estudiantes a cuestionar la formación de sus subjetividades en el contexto de las avanzadas formaciones capitalistas con la intención de generar prácticas pedagógicas que sean no racistas, no sexistas, no homofóbicas y que estén dirigidas hacia la transformación del orden social general en interés de una mayor justicia racial, de género y económica (p. 270).

Tomando en cuenta esta fundamentación, se puede inferir cómo este enfoque teórico de educación permite generar la posibilidad de espacios para el fortalecimiento de los derechos del conjunto de estudiantes y es, a la vez, la base para poder analizar si el modelo educativo costarricense presenta la capacidad de permitir la liberación ante las estructuras de opresión; es decir, si contribuye o no, al mejoramiento de la sociedad. 
La principal contradicción en esa línea implica la posibilidad de analizar cómo la educación, que es uno de los medios llamados a propiciar escenarios de respeto de la dignidad humana, ocasione un efecto contrario; es decir, cuando la educación genera opresión, adoctrinamiento o niveles de dependencia que violenten la dignidad humana que está llamada a desarrollar.

Metodológicamente, la educación desde el enfoque de derechos permite el análisis del discurso oficial detrás de la educación misma. Es vital empezar a construir espacios en donde los derechos humanos no sean solo un contenido teórico, que debe ser conocido por parte del grupo de estudiantes, sino que además debe promoverse su práctica en las aulas, a través de acciones cotidianas, promovidas en las instituciones de formación. A partir de este elemento, es vital establecer una reflexión en torno al tipo de educación que se desarrolla en el sistema educativo costarricense, y la necesidad de una formación orientada más hacia la transformación de la realidad vivida por la persona menor de edad.

El segundo elemento que aporta la Filosofía de la Liberación a la discusión que se plantea en este apartado consiste en el reconocimiento de las personas menores de edad como una población oprimida, lo cual implica la comprensión de aquellos elementos que son los que permiten tal proceso de sumisión. La búsqueda de la liberación implica establecer un análisis en donde se describa la realidad vivida por los y las estudiantes y la posible necesidad de transformar aquellos escenarios en donde se violenten sus derechos.

Hoy la situación de América Latina aparentemente ha empeorado con respecto a 1971. En esa fecha nació -en Argentina- la Filosofía de la Liberación Latinoamericana, a partir de la conciencia de la injusticia estructural que entonces oprimía a las mayorías populares de nuestro continente. Pues bien, hoy la exclusión se muestra más injusta y creadora de más víctimas, y se experimenta una globalización promovida según la ideología neoliberal que se autoproclama como pensamiento único. Este discurso parece dejar menos fisuras para alternativas que la Guerra Fría de aquel tiempo.

La moción a favor de las personas menores de edad, desde la lógica expuesta en esta reflexión, implica abandonar el adultocentrismo y tomar en cuenta al otro y la otra, en este caso a las personas menores de edad, en donde son ellos y ellas, desde su realidad y vivencia dentro del proceso educativo, quienes deberían ser la base desde la cual se vislumbre el posible aporte que el sistema educativo debe desarrollar para el fortalecimiento de sus derechos.

De forma operativa, la manera en que se lograría este cometido es empezando a evidenciar la importancia de la participación que las personas menores de edad deben tener en la sociedad misma, dentro del sistema educativo 
y en la institución escolar. Es vital establecer los mecanismos para darles voz y escucharlos desde su realidad, de manera que con ello se permita identificar aquellos aspectos sobre el papel que la educación cumple en su desarrollo integral, lo cual que se convierte en la fuente de información más rica de dicho proceso.

En este sentido y tomando como base la epistemología de la FL, es vital establecer una relectura del sistema educativo costarricense desde la perspectiva de la opción preferencial por los oprimidos, en donde se busque develar la situación de los derechos del conjunto de estudiantes.

El tercer y último aporte de la FL a la realidad educativa costarricense corresponde a la categoría del primado de la praxis, el cual es analizado por Ellacuria (1977) bajo los siguientes términos:

(...) por su carácter transformador, la praxis histórica es el ámbito donde más claramente se expresa la imbricación entre hombre y mundo, pues en ella las relaciones sujeto-objeto no son siempre unidireccionales, tanto que es preferible hablar de respectividad codeterminante (p. 57).

La Filosofía de la Liberación puede definirse como una filosofía que propugna la urgencia de la praxis histórica en orden a la justicia, pues se propone reflexionar acerca de las condiciones de dominación de los oprimidos, así como la estrategia práxica a seguir para superar dicha situación de postración.
Dussel (1972) concibe, desde una perspectiva personalista, tanto la praxis (la acción) como la poiésis (la producción en el trabajo del ser humano). A la primera, la describe como relación interpersonal, de forma que no hay relación persona a persona que no esté de alguna forma en actitud práxica, y la segunda es descrita como la relación persona-naturaleza.

El punto de partida de la Filosofía de la Liberación está, como el de toda filosofía, en la realidad y en la experiencia humana integral y radical de esta. Pero le es específico que se trate, ante todo, de la realidad histórico-social de las víctimas de la injusticia y la inhumanidad, como de hecho se ha dado históricamente en América Latina. Pues ellas interpelan radicalmente al filósofo no solo como hombre y como mujer, sino como filósofo, de modo que este responde con su praxis ético-histórica de liberación, incluida principalmente su praxis teórica específicamente filosófica.

Pues, ante el punto de partida del filosofar moderno en el "ego cogito" y su voluntad de poder, dicha filosofía lo puso desde el principio en el oprimido, el marginado, el pobre, en su trascendencia o exterioridad al sistema de opresión, y en su novedad histórica con respecto a esta. De ahí que no solo el filósofo como persona, sino que también su mismo filosofar, debe responder a la interpelación ético-histórica de los excluidos, con una opción por ellos y ellas, por su liberación humana integral y por la justicia hacia y con ellos y ellas. 
Hoy se trata no solo del pobre como oprimido, sino como excluido de la vida y convivencia dignas $y$, no pocas veces, de la vida y convivencia a secas. Dicha opción por los excluidos es, sin embargo, universalmente humana, porque es de hecho la opción por lo humano y la dignidad humana como tal. De ahí que dicha situación y opción se constituyan entonces en un lugar hermenéutico del filosofar. Se trata del lugar desde donde se interpretan y disciernen no solo la actualidad histórica latinoamericana, sino también toda la realidad sin más, en este caso, la realidad educativa.

Así es como se replantean, todo el asunto y todos los asuntos del pensar, no solo los referentes a las cuestiones sociales o a los oprimidos y oprimidas. Dicha opción por los excluidos y excluidas (víctimas históricas) es universalmente humana, ya que se trata precisamente de la opción por lo humano integral, de todos los hombres y mujeres, ante la deshumanización de las mayorías e, indirectamente, de todos y todas.

Ahora bien, una vez alcanzado un concepto tan abarcador de la praxis histórica, salta a la vista que la teoría en cuanto momento de esa praxis o, más radicalmente, la inteligencia humana misma, queda afectada por la historicidad. Dar cuenta de esto supone replantear adecuadamente el viejo problema filosófico del conocimiento desde el punto de vista de su historicidad. Para ello se necesita, según Ellacuría (1977, s.p.), una "interpretación de la inteligencia como inteligencia histórica".
La inteligencia histórica es, por lo pronto, una inteligencia situada, es decir, una inteligencia que sabe no poder entrar al fondo de sí misma más que situadamente y pretendiendo entrar al fondo de la situación tomada en su totalidad. Dicho en otros términos, la realidad es histórica y solo un logos de la historia, un logos histórico, puede dar razón de la realidad. Un logos puramente natural nunca daría razón adecuada de una realidad que es más que naturaleza.

La forma de adaptar tal principio en el sistema educativo costarricense se justifica a partir de dos aspectos centrales. El primero de ellos es partir de que la realidad educativa es histórica, desde donde los derechos humanos son vivenciados, una realidad que es vivida por cada uno del grupo de estudiantes, cuerpo docente y los profesionales del Ministerios de Educación Pública, todos juntos permiten la ejecución de los programas y planes de estudio, lo cual expresa los componentes de la realidad educativa en su totalidad. El segundo aspecto de la praxis en la realidad educativa costarricense hace referencia a la necesidad de elaboración de propuestas que promuevan el desarrollo de la participación estudiantil, como un medio de expresar el primado de la praxis, a favor de la persona menor de edad, quien debe ser entendida como la oprimida. Se debe hacer un esfuerzo de intervenir en esa realidad para transformarla, de ahí la búsqueda de espacios de participación para el grupo de estudiantes que permitan el fortalecimiento de sus derechos y el respeto de su dignidad. 
El esfuerzo que se presentan en estas líneas implica la posibilidad de darle un sustento no solamente teórico, sino filosófico a los derechos humanos en el sistema educativo costarricense. Es un compromiso ético, en cuanto implica una forma de concebir la realidad social, al tiempo que permite no solamente la generación de nuevos conocimientos y aportes para la academia, sino que se convierte en un espacio que intente cambiar y transformar desde lo mínimo, las relaciones sociales entre el mundo del adulto y la persona menor de edad, en la esfera de la institución escolar desde donde se gesta el desarrollo de la autonomía del grupo de estudiantes.

Finalmente, implica un posicionamiento que atraviesa cada una de las líneas que forman parte del análisis, en donde no solo se busca coherencia teórica metódica, sino que permite darle sentido y razón a la práctica de la educación desde el enfoque de derechos, orientándole una razón de ser y un compromiso por los Derechos Humanos de los y las Adolescentes, los y las niñas.

\section{Referencias}

Aguiló, A. J. (2009). Los Derechos Humanos como campo de luchas por la diversidad humana: Un análisis desde la sociología crítica de Boaventura de Sousa Santos. Revista universitas humanística. 68, julio-diciembre de 2009, pp. 179-205. Bogotá, Colombia ISSN 0120-4807. [En línea] Disponible en http://revistas.javeriana.edu.co/index.php/univhumanistica/ article/view/2272
Alarcón, N. y Molina, L. (2008). La Desigualdad Social: Desarrollo y Desafíos del Trabajo Social desde la Reconceptualización en América Latina. Revista Palabra, 9. Recuperado de: http://revistaseletronicas.pucrs.br/ojs/index.php/fass/article/ viewFile/1044/3231

Arrollo, R. (2009). La Dimensión histórica de los Derechos Humanos: movimiento y enfoque teórico metodológico. Conferencia Inaugural del curso de Inducción. Universidad de Costa Rica. Sábado 8 de Agosto del 2009.

Dussel, E. (1972). Para una ética de la liberación latinoamericana. Argentina: Editorial Siglo XXI.

De Lemos, L. (2007). A gente precisa é trabalhar ser criança nas políticas dirigidas às crianças. [La gente que trabaja con niños y las políticas para la infancia]. Posgrado en Trabajo Social. Universidad Federal y Centro Socioeconómico, Belén. Recuperado de: http://livros01.livrosgratis.com. br/cp051757.pdf

De Sousa. (2004). La Educación Latinoamericana en el Siglo XXI: escenarios hacia las pedagogías de la alienación, de la domesticación y de la transformación. Recuperado de http://www.apse.or.cr/webapse/pedago/ enint/souza04.pdf

Ellacuría, I. (1973). Teología política. San Salvador: Ediciones del Secretariado Social Interdiocesano.

Ellacuría, I. (1977). Función liberadora de la filosofía. San Salvador: Editorial UCA.

Ellacuría, I. (1982). Universidad, Derechos Humanos y Mayorías Populares. Estudios Centroamericanos (ECA), 406. 
Recuperado de http://www.uca.edu.sv/ facultad/chn/c1170/Universidad, $\% 20$ derechos $\% 20$ humanos $\% 20 y \% 20$ mayorias\%20populares.pdf

Fanlo, L. (s.f.). Los derechos del niño y las teorías de los derechos: introducción al debate. Antología del curso Enfoques teóricos para la comprensión e intervención en las situaciones de la niñez, la adolescencia y la familia. Universidad de Costa Rica, 2012.

Fernández, A. (2007) Reconsideraciones en torno de los derechos de la niñez y la adolescencia. Revista KAIROS. Revista de Temas Sociales. Año 11. № 20. Recuperado de: http://www.revistakairos.org/ k20-archivos/FHassan.pdf

Herrera, J. (Ed.). (2000). El vuelo de Anteo: Derechos Humanos y Crítica de la Razón Liberal. Bilbao: Desclée de Brouwer.

Lukács, G. (1960). La crisis de la filosofía burguesa. Buenos Aires: La Pléyade.

McLaren, P. (1997). Pedagogía crítica y cultura depredadora. Barcelona: Paidós.
Molina, C. (s.f.). Ignacio Ellacuría: filosofía y liberación en América Latina. Recuperado de: http://www.uca.edu.sv/filosofia/ admin/files/1201492447.pdf

Mora, J. (2006). Sobre los inéditos de Ignacio Ellacuría. Revista Internacional de Pensamiento Político, I Época, 2, pp. 181-187. Recuperado de: http://www.pensamientopolitico.org/Descargas/RIPP02177183. PDF

ONU (1948). Declaración Universal de los Derechos Humanos. Recuperado de www. un.org/es/documents/udhr/

Orozco, J. (s.f.). Los Derechos Humanos y la polémica entre Isnaturalismo y iuspositivismo. Recuperado de http://biblio.juridicas. unam.mx/libros/1/399/3.pdf

Samour, H. (s.f.). Zubiri y la filosofía de la liberación. Recuperado de http://www. uca.edu.sv/facultad/chn/c1170/samour2. html\#_ednref25

Recibido: 19/11/2014 • Aceptado: 27/8/2015 